\title{
Explorando tecnologias de IoT no contexto de Health Smart Home: uma abordagem para detecção de quedas em pessoas idosas.
}

\author{
Leandro Yukio Mano \\ Instituto de Ciências Matemáticas e de Computação \\ Universidade de São Paulo \\ São Paulo, São Paulo, Brasil. \\ Marcio Maestralo Funes \\ Instituto de Ciências Matemáticas e de Computação \\ Universidade de São Paulo \\ São Paulo, São Paulo, Brasil.
}

\begin{abstract}
Currently, it is increasing the number of patients who are treated at home, especially in countries like Japan, United States and European countries. In addition, the number of elderly has increased significantly in the last $\mathbf{1 5}$ years, and these people prefer to receive medical treatment in their homes. However, critical situations can happen during this recovery time, e.g., the elderly patient suffering a fall and aggravate the situation. In this scenario, advances in Ubiquitous Computing and Internet of Things (IoT) have contributed to avoid these situations. In particular, embedded devices, along with the motion capture through sensors can be applied to develop solutions that offer greater safety for patients who receive treatment at home. However, there is a difficulty to classify an individual's movements and identify, in fact, a move considered unusual. Thus, the main objective of this work is the detection and classification of movements using data sets of different sensors and embedded device. Paradigms of Artificial Intelligence (AI) were applied to the classification of the movements and tests performed in architecture SAHHc. The results showed a 96.62\% precision in identifying the activities performed by a patient/elderly.
\end{abstract}

Keywords: Internet of Things, embedded systems, elderly, healthcare.

Resumo - Atualmente, é crescente o número de pacientes que são tratados em casa, principalmente em países como o Japão, Estados Unidos e da Europa. Além disso, o número de idosos tem aumentado significativamente nos últimos quinze anos, e essas pessoas, muitas vezes, preferem receber tratamento médico em suas residências. No entanto, podem acontecer situações críticas durante esse período de recuperação, como por exemplo, o paciente idoso sofrer uma queda e agravar o seu quadro clínico. Neste cenário, avanços em Computação Ubíqua e Internet das Coisas (IoT) têm contribuído para evitar essas situações. Em particular, dispositivos embarcados, juntamente com a captura de movimentos por meio de sensores, podem ser aplicados para desenvolver soluções que ofereçam mais segurança para essas pessoas. Todavia, observa-se uma dificuldade de classificar os movimentos de um indivíduo e identificar, de fato, um movimento considerado anormal. Assim, o principal objetivo deste trabalho é a detecção e classificação dos movimentos utilizando dados conjuntos de sensores distintos e dispositivo embarcado. Paradigmas de Inteligência Artificial (AI) foram aplicados para a classificação dos movimentos e os testes realizados na arquitetura SAHHc. Os resultados apontaram uma precisão de $96.62 \%$ na identificação das atividades executadas por um paciente/idoso.

\author{
Tiago Volpato \\ Instituto de Ciências Matemáticas e de Computação \\ Universidade de São Paulo \\ São Paulo, São Paulo, Brasil. \\ José Rodrigues Torres Neto \\ Instituto de Ciências Matemáticas e de Computação \\ Universidade de São Paulo \\ São Paulo, São Paulo, Brasil.
}

\section{INTRODUÇÃO}

$\mathrm{O}$ número de pessoas idosas tem crescido rapidamente em vários países, incluindo os EUA e o Brasil [1] [2]. Além de serem mais propensas a doenças, essas pessoas têm a tendência de morarem sozinhas [3], seja por motivos culturais, familiares, entre outros. Além disso, é mais cômodo para as pessoas da terceira idade receberam tratamento de saúde domiciliar, seja após um procedimento cirúrgico ou depois de receberem alta do hospital, por exemplo. Dessa forma, são necessárias estratégias para que o cuidado desses pacientes seja mais acessível, uma vez que nem todos podem custear um profissional da saúde por tempo integral. Neste cenário, soluções tecnológicas podem ser utilizadas para monitorar pessoas idosas e/ou adoecidas, que não conseguem tomar conta de si mesmas e precisam de cuidados constantes. Isso muitas vezes leva ao uso da tecnologia para monitorar seu cotidiano e/ou enquanto se recuperam em casa [4] [5].

A utilização da tecnologia nesse contexto é viável, principalmente em países onde o número de pessoas nessas condições é alto, podendo fornecer sistemas que possam monitorá-los e emitir alertas para a equipe médica e/ou familiares sempre que necessário. Diante disso, o uso de tecnologias que possibilitem monitorar a saúde das pessoas torna-se extremamente relevante [5]. Assim, o conceito de Health Smart Homes (HSH) emerge da combinação de Telemedicina, Internet of Things (IoT) e Sistemas de Informação. Uma HSH, pode ser definida como uma casa inteligente e equipada com dispositivos especializados para os cuidados da saúde à distância, incluindo smartphones e tecnologias vestíveis (por exemplo, smartwatch). Tais dispositivos podem tomar ações sempre que uma situação crítica é detectada. Devido a essas características, uma HSH permite a criação de um ambiente onde as pessoas com algumas dificuldades e/ou doenças, gozem de maior independência e segurança em suas rotinas diárias. Algumas iniciativas de trabalhos que exploram esse conceito são encontradas em [4] [6] [7].

Durante nossa pesquisa, verificou-se que a maioria dos estudos estão concentrados em melhorar a vida diária de pacientes, fornecendo-lhes aparelhos com funcionalidades 
específicas, tais como alerta de alarmes [8] [9] [10]. Em geral, esses trabalhos propõem a detecção de movimentos dos pacientes por meio de sensores, por exemplo, giroscópio e/ou acelerômetro. No entanto, observa-se a dificuldade de classificar esses movimentos como normais ou como anormais (uma queda, por exemplo). Assim, algoritmos de Aprendizado de Máquina, do inglês Machine Learning (ML), foram aplicados nos dados capturados pelos sensores citados acima, de forma conjunta, para categorizar os movimentos e obter uma melhor acurácia na detecção de uma possível queda de pessoas idosas, fornecendo-lhes um maior grau de conforto durante o cuidado em casa.

Nossa proposta de trabalho tem a hipótese de que a utilização da abordagem baseada em múltiplos sensores (acelerômetro e giroscópio), permite a análise das seguintes atividades: correndo, andando, sentado, em pé, deitado e queda. A abordagem proposta é realizada pelos indivíduos de maneira não intrusiva. $\mathrm{O}$ trabalho difere dos estudos citados anteriormente e permite novos recursos para a qualidade de vida e cuidado das pessoas. Ainda, a abordagem proposta investigará a identificação dos padrões do cotidiano e estratégias de aprendizado das atividades e situações, de modo que este possa fornecer um feedback da atividade realizada pelo indivíduo em tempo de execução. Portanto, este projeto tem como objetivo desenvolver e avaliar um modelo que permita: i.) identificar a atividade realizada do usuário; ii.) prover um mecanismo que possa monitorar de maneira inteligente e não intrusiva as atividades do cotidiano do usuário, e; iii.) explorar uma abordagem para classificação com a utilização dos conceitos de IoT.

O restante deste texto está organizado da seguinte forma: a Seção 2 contempla a aplicação de tecnologias IoT para cuidados da saúde em casas inteligentes. A Seção 3 apresenta a arquitetura SAHHc em que a proposta é baseada. Em seguida, a Seção 4 descreve os conceitos para a Saúde em Casas Inteligentes, abordando com mais detalhes nossa proposta para detecção de quedas, utilização de sensores e dispositivos embarcados. A Seção 5 apresenta os resultados dos experimentos realizados, tanto na identificação e classificação das atividades realizadas pelo idoso/paciente quanto do processamento e comunicação dos dados na arquitetura. Por fim, a Seção 6 apresenta algumas observações finais e aponta possíveis direções futuras para este estudo.

\section{INTERNET DAS COISAS APLICADA À SAÚDE EM CASAS INTELIGENTES}

Esta seção examina os avanços feitos em HSH baseado em tecnologias IoT, com o objetivo de apresentar o que tem sido desenvolvido e os principais desafios desta área de pesquisa.

Internet of Things, é um fenômeno tecnológico proveniente de empreendimentos inovadores e conceitos em tecnologia de informação e comunicação associados com conectividade em Computação Pervasiva, Computação Ubíqua e Ambiente Inteligente [11]:

- Computação Pervasiva: implica que o computador está embarcado no ambiente de forma invisível para o usuário. Este conceito permite que sistemas computacionais tenham a capacidade de obter informações do ambiente ao qual estão inseridos e, dinamicamente, executar ações para atender dispositivos e usuários;

- Computação Ubíqua: utiliza os conceitos de pervasividade aliados ao conceito de mobilidade. Onde o sistema ubíquo pode estar em todo lugar de forma invisível ao usuário (pervasivo), mas não restringe o seu campo de ação a um só ambiente, uma vez que pode estar em movimento constante juntamente com o usuário (mobilidade);

- Inteligência Ambiental: significa a capacidade de os objetos registrarem as mudanças no ambiente físico e, assim, interagir ativamente em um processo.

Normalmente, os objetos que cumprem esses requisitos são chamados de "objetos inteligentes" [12]. Assim, a IoT é definida como a construção das redes de "coisas" interoperáveis, isto é, a capacidade de objetos inteligentes comunicarem entre si.

Ainda, outra descrição para IoT diz respeito à utilização de objetos para construir uma rede dinâmica conectada pela Internet [13], tornando-a um espaço virtual on-line para um sistema embutido no mundo físico real. IoT possui algumas características típicas, que são: i.) criação de novas redes independentes que operam com suas próprias infraestruturas; ii.) implementação de novos serviços e; iii.) aplicação de novas e diferentes modalidades de comunicação entre pessoas-coisas e coisas-coisas [14] [15]. Os dispositivos inteligentes permitem o funcionamento da IoT, que podem estar com seu status ativo (na tomada de decisão local) ou passivo (no armazenamento de dados do sensor para leitura). Sendo assim, uma das principais razões para o uso de IoT é o fornecimento de informações relevantes quando e onde for necessário, além de ser capaz de preencher a lacuna entre a Web e o mundo real [15].

Nesta perspectiva, uma proposta de aplicação dos conceitos de IoT surge para HSH, onde sistemas computacionais são aplicados para apoiar as pessoas idosas e pessoas com necessidades especiais em sua rotina diária. Helth Smart Home pode ser definida como uma casa (ou ambiente) inteligente equipada com dispositivos eletrônicos especificamente projetados para cuidados com a saúde, controlados por intermédio de um Remote Control Center (RCC) [7]. Conforme ilustra a Figura 1 o RCC garante a resposta em caso de emergência e promove uma interface entre o paciente, que está em casa, com um conjunto de pessoas envolvidas no processo de cuidados com a saúde, tais como familiares, médicos ou serviço de emergência e hospital [7].

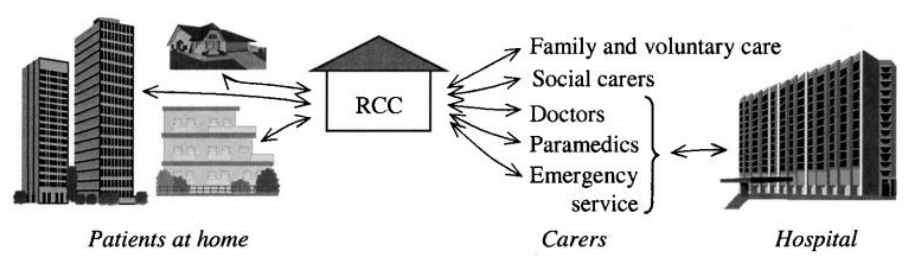

Figura 1: Visão geral de um cenário HSH [7]. 
O principal objetivo de uma HSH é manter e promover a independência dessas pessoas e, assim, aumentar a segurança no seu estilo de vida e no seu ambiente doméstico. A necessidade para tais aplicações surge da evolução demográfica nos países industrializados, onde a expectativa de vida da população aumenta, enquanto que a taxa de natalidade está em declínio. Estas circunstâncias exigem soluções mais acessíveis para manter os gastos com saúde de acordo com a possibilidade econômica das pessoas [14]. Aplicações HSH incluem serviços, produtos e conceitos para aumentar a qualidade de vida, bemestar e segurança das pessoas, beneficiando o indivíduo, a economia e a sociedade [14] [16]. Assim, os campos de necessidades de pessoas com alguma limitação e/ou idosas em todas as aplicações são: i.) Saúde; ii.) Segurança; iii.) Paz de espírito; iv.) Independência; v.) Mobilidade; e vi.) Interação Social.

Neste sentido, algumas obras já foram propostas. O trabalho realizado por [7] revisa o conceito emergente de $\mathrm{HSH}$, retratando potenciais aplicações desses sistemas, tais como sistemas de alarme social e monitoramento remoto. $\mathrm{O}$ estudo também analisa tecnologias proeminentes que estão sendo implantadas e relata projetos pilotos de sistemas que já estão sendo utilizados em hospitais e residências. Os avanços dos cuidados da saúde no contexto domiciliar se devem também à aplicação de Ambientes Inteligentes (Aml) [17] [18] e Artificial Intelligence (AI) [19]. A combinação de modernos equipamentos de detecção com avançadas técnicas de processamento de dados e redes sem fio possibilita a criação de ambientes digitais que melhoram a vida diária dos pacientes que recebem cuidado em domicílio.

Estudos na literatura [5] [20] são focados no uso de sensores próximo ao corpo e dispositivos específicos ao prover assistência para pessoas, em particular, idosos que precisam de cuidados com a saúde. [20] propõem o uso de câmeras para melhorar a análise do ambiente (por exemplo, detectar possíveis situações de risco), proporcionando um sistema que não seja intrusivo. Em [5], é apresentada uma arquitetura para uma rede de área pessoal sem fio (WPAN), que aproveita os benefícios oferecidos por um ambiente inteligente utilizando dados de diferentes sensores. O objetivo do sistema é garantir a segurança e fornecer serviços aos usuários através de técnicas de monitoramento com base no processamento de imagem, que pode se integrar com outros dispositivos de controle. Neste caso, um sensor baseado em processamento de imagem tem uma combinação de filtros, comparações de taxa de quadro e outros algoritmos, que atuam de forma inteligente no ambiente. Isso permite a detecção de movimentos e padrões nas atividades de um paciente, como por exemplo a velocidade e a direção que ele se movimenta.

Outra iniciativa de trabalho que usa tecnologias de IoT para melhorar o cuidado de pacientes idosos pode ser encontrado em [21]. O estudo propõe um framework baseado em camadas que compreende a dependência e interconectividade de biosensores, canais de comunicação, microcontroladores, gateways, Internet e aplicativos para o monitoramento.

Em outra perspectiva, o trabalho [20] apresenta uma solução para reduzir os acidentes que pessoas idosas podem sofrer durante a noite. $\mathrm{O}$ sistema baseia-se na visão noturna e opera com o mínimo de interações com esses pacientes. Através de algoritmos baseados na noção de causalidade, raciocínio espaço-temporal e alertas, o sistema fornece informações para os usuários sobre acidentes ou possíveis situações de riscos que o paciente idoso possa sofrer. Os resultados do sistema (alertas e explicações) são baseados em um conjunto de regras causais que se referem aos locais e horário das atividades do idoso, levando a um provável cenário de risco dentro de casa.

Outro importante trabalho foi desenvolvido pelo Laboratório de Computação Mobile e Pervasiva da Universidade da Flórida. O Gator Tech Smart House (GTSH) [22] é um laboratório experimental e um ambiente real destinado a validar tecnologias e sistemas para casas inteligentes. O objetivo do GTSH é criar ambientes que auxiliem seus residentes e sejam capazes de mapear o mundo físico com monitoramento remoto e serviços de intervenção, conduzindo pesquisas e desenvolvendo atividades projetadas para assistência aos idosos e pessoas com necessidades especiais, aumentando sua independência e contribuindo para uma melhoria na qualidade de vida. No mesmo sentido, o Instituto de Tecnologia da Georgia desenvolveu o Aware Home Research Initiative (AHRI) [23]. A pesquisa investiga como as novas tecnologias podem impactar a vida das pessoas no ambiente domiciliar em três áreas principais: i.) saúde e bem-estar; ii.) meios de comunicação e entretenimento digital, e; iii.) sustentabilidade. $\mathrm{O}$ trabalho permitiu a pesquisa em muitas áreas, tais como fornecimento de dados para residências, sensoriamento inovador, controle de infraestrutura e uma variedade de aplicações para ajudar os moradores.

Como pode-se notar, o escopo de aplicações nestes domínios é muito amplo. Por esta razão, ambientes HSH são estruturados em três níveis distintos: Hardware (sensoriamento, redes sem fio), Middleware (captura de dados, segurança de dados, integração de TI) e Serviços (processamento de sinais biológicos, processos centrados em aplicações, serviços) [24]. Um cenário HSH é caracterizada por estarem conectados, sensível ao contexto pessoal, adaptável e de antecipação. O que é suposto, ser capaz de fornecer todas as características necessárias para um ambiente assistido. No que diz respeito aos campos de necessidades para as pessoas com alguma deficiência, mobilidade reduzida e idosas, é possível abranger todos os campos através da IoT. O monitoramento de doenças crônicas (saúde), disposição com alimentos frescos e sistemas de alarme (segurança), serviços de lembrete (paz de espírito) e permitir a comunicação pessoa-a-pessoa, por exemplo, com os parentes (interação social) sem perceber a tecnologia por trás dele são apenas algumas aplicações mencionáveis de HSH através da IoT. A comissão da União Europeia incentiva essa hipótese, dizendo: "O escopo de aplicações da Internet das Coisas é esperado para contribuir grandemente a abordar 
desafios sociais de hoje" [25]. Nesse contexto, este trabalho abrange conceitos de IoT em HSH no sentido de detectar possíveis quedas de pacientes idosos utilizando dados conjuntos de sensores de movimento, que serão classificados utilizando um dispositivo embarcado.

\section{DISCUSSÃO SOBRE O NOSSO AMBINETE BASEADO EM IOT}

Nossa proposta para identificação de quedas de idosos é baseado no sistema chamado Smart Architecture para In-Home Healthcare (SAHHc) [4]. SAHHc baseia-se na utilização da IoT para o monitoramento inteligente e individualizado de pacientes idosos em suas casas. As principais características do SAHHc são a escalabilidade de seus componentes e a capacidade de inferir as emoções ou sentimentos da experiência do paciente (como a dor ou o início da depressão), aliados com acompanhamento individualizado.

SAHHc é composto por dois elementos: i.) Sensores e; ii.) o Nó Decisor. Os elementos sensores são dispositivos distribuídos no ambiente; eles podem ser encontrados em grandes números e o seu objetivo é recolher informação (por exemplo, capturar imagens ou movimentos) sobre a saúde do paciente e enviá-lo para o elemento Nó Decisor. O elemento Nó Decisor processa as informações recebidas e toma decisões que visam preservar a saúde dos pacientes ou dar-lhes um tratamento adequado. Inicialmente, o elemento Nó Decisor é definido como um único nó (uma máquina) que também trabalha como gerente de rede, mas suas funções podem ser divididas entre vários tomadores de decisão para fins de escalabilidade. Além disso, o Nó Decisor serve como uma interface entre o paciente e o cuidador e/ou familiar. A Figura 2 ilustra os elementos em um cenário onde o sistema SAHHc proposto é aplicado.

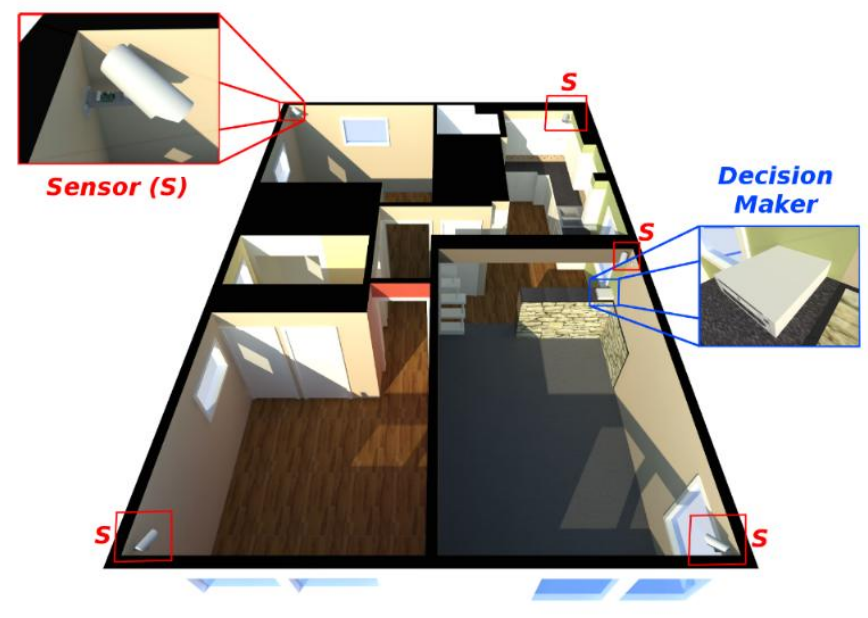

Figura 2: Cenário de operação do SAHHc [4].

Dadas as características do ambiente onde este tipo de aplicação pode ser implementado, os dispositivos, em grande maioria, devem ser executados integrando-se com a energia do ambiente e/ou usar a bateria como fonte de backup. Além disso, presume-se que o ambiente tem uma rede de comunicação interna que pode ser utilizado pelo sistema.

Embora as redes sem fios possam assegurar a aplicação do SAHHc para facilidade e mobilidade, uma rede com fios também pode ser

utilizada.

A Figura 3 apresenta os elementos configuráveis (níveis) da arquitetura SAHHc. O SAHHc é dividido nos seguintes níveis:

- Nível 0 (com os dispositivos embarcados): realiza o reconhecimento, pré-processamento dos dados monitorados pelo sensor;

- Nível 1 (servidor local): realiza o monitoramento da casa e do paciente. Nesse sentido, é necessário ter maior poder de processamento e disponibilidade de armazenamento. Este nível também é responsável por executar todo o controle do sistema;

- Nível 2 (servidor remoto): o processamento dos dados na nuvem é realizado quando o servidor local está sobrecarregado e desequilibrado. Assim, ele fornece um esquema de balanceamento de carga e garante a escalabilidade da arquitetura SAHHc.
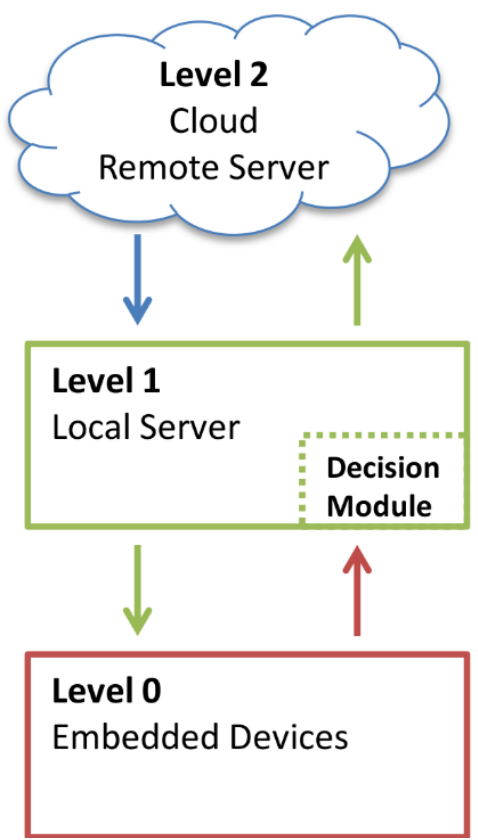

Figura 3: Elementos configuráveis (níveis) da arquitetura SAHHc [4].

As características da arquitetura SAHHc não são firmemente acopladas e isso permite configurá-las de acordo com necessidades específicas. Este tipo de flexibilidade garante que os novos elementos possam ser facilmente conectados à arquitetura sempre que necessário. Por exemplo, se o paciente começa a usar novos dispositivos (tais como, tablets, smartphones e smartwatches) que são capazes de coletar informações sobre seu comportamento; assim, estes novos dispositivos podem ser instanciados no sistema. Se o novo dispositivo é capaz de recolher um novo tipo de dado do paciente, também é necessário que o Nó Decisor possa receber uma instância para o processamento deste tipo de dado e, even- 
tualmente, compor esses dados com outras informações que já estão sendo utilizadas. Nesse sentido, o trabalho proposto pretende utilizar dispositivos móveis, tanto smartphones ou tecnologias vestíveis, para a aquisição de novos dados. Nesse contexto, os dispositivos possuem sensores que podem ser úteis para o monitoramento de pacientes, por exemplo, o acelerômetro e giroscópio para a detecção de um acidente ou comportamento anormal.

\section{ACOMPANHAMENTO INTELIGENTE DO IDOSO PARA HEATH SMART HOMES}

Visão computacional é uma abordagem utilizada em diversos cenários de acompanhamento inteligente para idosos, uma vez que permite a aquisição de quantidades enormes de dados multidimensionais, relacionados com o ambiente e o indivíduo monitorado. Essa abordagem pode ser utilizada para "aprender" as atividades da vida diária de pessoas idosas e analisar problemas de saúde ou perturbação cognitiva [26].

$\mathrm{Na}$ atual proposta, a Visão Computacional fornece uma camada difundida com o idoso, e permite o monitoramento não-intrusivo. Além disso, a arquitetura pode fazer uso de outros dispositivos de informação para melhorar a precisão dos dados atualmente disponíveis ou fornecer melhores formas de comunicação para o indivíduo.

A.Acelerômetro e Giroscópio para monitoramento de pessoa

Muitos sistemas têm utilizado o monitoramento dos movimentos realizados pelos usuários em suas residências como uma forma de monitorar sua rotina a distância e de modo não intrusivo (Figura 4). Para que atue de forma não intrusiva, o sistema apenas promove alguma ação quando detecta alterações no padrão de movimentos que um usuário normalmente efetua, portanto, a captura de movimentos precisos e de forma constante é indispensável.

Neste sentido, o acelerômetro e o giroscópio são explorados, de forma conjunta ou separada, frequentemente no contexto de HSH devido a precisão que podem fornecer nos dados de movimento capturados e por possuírem um caráter não intrusivo. Assim, pode-se entender suas diferenças da seguinte forma:

-Acelerômetro: sensor de movimento que captura a variação na aceleração que um corpo exerce no espaço de forma tridimensional, ou seja, nos eixos $\mathrm{X}, \mathrm{Y}$ e Z [27]. Por meio desses valores, é possível determinar o deslocamento de um corpo em movimento.

- Giroscópio: sensor de movimento que captura a mudança de rotação que um corpo exerce no espaço de forma tridimensional, ou seja, nos eixos $\mathrm{X}, \mathrm{Y}$ e $\mathrm{Z}$ [28]. Por meio desses valores, é possível determinar a rotação e a mudança de direção de um corpo em movimento.

Como exemplo de aplicação de acelerômetro e giroscópio, o serviço QMedic - Medical Alert System oferece aos seus usuários uma pulseira com um botão que pode ser acionado sempre que o usuário precisar de assistência médica (como ilustrado na Figura 4). Além de monitorar quedas, o serviço captura os movimentos do usuário de forma passiva antes de executar uma ação [29].

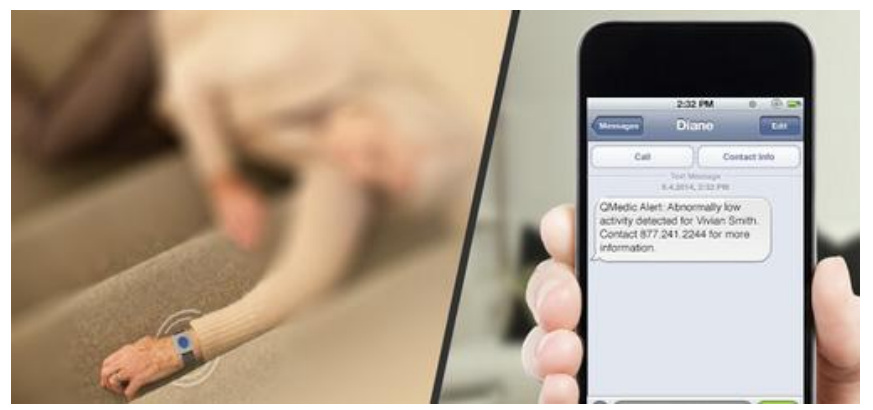

Figura 4: Exemplo de aplicação de sensor para monitoramento [29].

Essa ação pode ser o envio de informações para um dispositivo móvel, tais como mensagens de texto, informando sobre uma situação de risco sofrida pelo paciente.

\section{B.Inteligência Artificial para detecção de quedas}

A abordagem proposta aplica-se a identificação de quedas/acidentes por meio do monitoramento do idoso. Isto é realizado através do emprego de paradigmas de AI distintas, como k-Nearest Neighbor, Árvore de Decisão, Redes Bayesianas, Support Vector Machine e Multi Layer Perceptron [30]. A seguir, encontra-se uma súmula conceitual dos algoritmos de classificação utilizados no reconhecimento do estado do usuário:

•k-Nearest Neighbor classification (kNN) - Classificação dos $k$-vizinhos mais próximos. O $\mathrm{kNN}$, utilizado em alguns trabalhos [31] [32], é uma abordagem de classificação nãoparamétrica, isto é, não assume nenhuma distribuição de probabilidade para os dados a priori. O algoritmo kNN encontra um grupo de $k$ objetos no conjunto de treino que estão mais próximos do objeto de teste e o classifica com a classe predominante dos seus $k$-vizinhos [33].

-Decision or Regression Tree - A árvore de decisão ou regressão, aplicada nos trabalhos de [31] e [34], utiliza-se de uma árvore para realizar a classificação ou a estimação de um valor de um dado teste. Um dos algoritmos mais famosos para gerar essas árvores é o C4.5, cuja árvore cresce a partir de uma abordagem "dividir e conquistar" e é ramificada utilizando o atributo que obtiver o melhor ganho de informação [35].

-Bayesian Networks - A Rede Bayesiana é um modelo de grafo probabilístico, aplicado aos trabalhos de [34], [36] e [37], e que representa um conjunto de variáveis aleatórias e suas dependências condicionais como um grafo acíclico direto [33]. Por exemplo, uma rede bayesiana pode representar a relação entre os sintomas e as doenças. Dados os sintomas, pode-se inferir a probabilidade de várias doenças. No caso desse trabalho em particular, dadas as características do acelerômetro e giroscópio pode-se inferir a probabilidade de determinada atividade.

- Support Vector Machine (SVM) - O algoritmo SVM, aplicados por [34], [36] e [38] para o reconhecimento de emoções, é um dos métodos mais robustos e acurados entre os algoritmos conhecidos. Segundo [33], o objetivo do SVM é encontrar a melhor função para distinguir duas ou mais classes 
dos dados de treinamento. A garantia da melhor função é atingida, pois o algoritmo maximiza a margem entre as classes e oferece melhor habilidade de generalização [36], [38] e [39].

-MultiLayer Perceptron (MLP) - A MLP é um modelo de AI que mapeia conjuntos de dados de entrada para um conjunto de saídas apropriadas aplicados em vários trabalhos na literatura [40] [41]. Um MLP é composto por várias camadas de nós em um grafo direcionado, com cada camada completamente conectado à próxima. Exceto para os nós de entrada, cada nó é um neurônio (ou elemento de processamento) com uma função de ativação não-linear. Nessas redes, cada camada tem uma função específica. A camada de saída recebe os estímulos da camada intermediária e constrói o padrão que será a resposta. As camadas intermediárias funcionam como extratoras de características, seus pesos são uma codificação de características apresentadas nos padrões de entrada e permitem que a rede crie sua própria representação, mais rica e complexa, do problema [42].

A área de classificação de dados visa construir sistemas que aprendam com os dados. No contexto de monitoramento de sinais, os algoritmos de classificação utilizam o tipo de aprendizado supervisionado. Os algoritmos de aprendizado supervisionado são treinados com exemplos rotulados, isto é, a saída da entrada é conhecida, por exemplo, uma imagem que contém uma face com expressão já conhecida [39] [43].

\section{C.Discussão sobre o nosso ambiente baseado em IoT}

A arquitetura SAHHc é baseado em INCAS (INCidentsAware System) [44]. O principal objetivo do INCAS é antecipar e detectar incidentes que possam ocorrer, bem como informar ao usuário, cuidador ou qualquer outra pessoa preocupada com o incidente/acidente no interior da casa. INCAS tem três módulos básicos - o módulo móvel, módulo de serviço Web e módulo sensor (Figura 5). O módulo móvel comunica-se com o módulo de serviço Web através da rede celular (por exemplo, 3G ou 4G) ou Wi-Fi. Uma aplicação Android configura as preferências do usuário, como por exemplo, a forma como ele gostaria de ser informado sobre um incidente. Estas opções de alerta podem envolver um áudio, vibração ou exibir uma imagem animada. Assim, o usuário pode escolher uma configuração que melhor se adapta a sua deficiência específica, por exemplo, visuais ou deficiência auditiva. Além disso, o aplicativo mostra ao usuário uma lista de ambientes que podem ser monitorados. Uma vez que um quarto foi selecionado para o monitoramento, por exemplo, uma mensagem é enviada para o módulo de serviço Web que armazena essas informações no banco de dados. Para evitar problemas de segurança, quando esta aplicação é iniciada, ele solicita uma autenticação do usuário e também todas as mensagens trocadas entre os módulos são transferidas por meio do Secure Socket Layer (SSL).

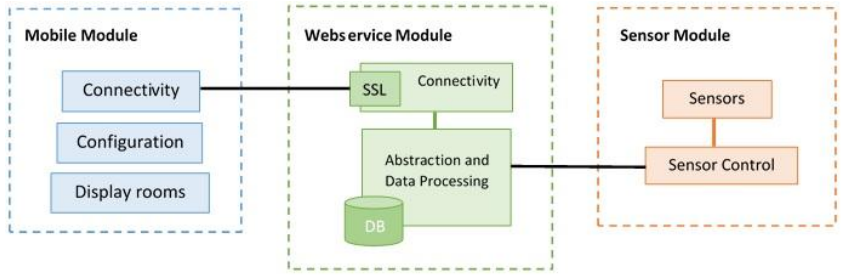

Figura 5: Extensão geral do INCAS [44].

O módulo de serviço Web está ligado a um servidor local na casa do usuário. Este módulo precisa de uma ligação com a Internet para receber pedidos a partir do dispositivo computacional utilizado pelo usuário (nesse caso, tecnologias vestíveis) e uma conexão sem fio via Wi-Fi para receber os dados recolhidos pelos sensores. Nesta fase, há um módulo de captação e processamento de dados, conforme ilustra a Figura 6. Assim, os dados são armazenados, analisados e inferidos e, portanto, em posição de saber se os eventos capturados pelos sensores representam uma queda real do usuário.

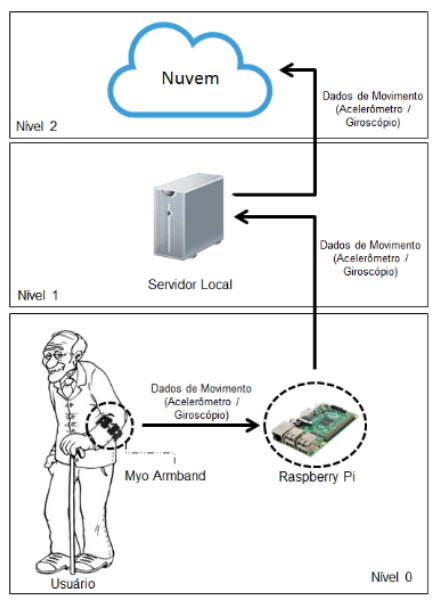

Figura 6: Módulo de captação e processamento de dados.

A arquitetura INCAS permite o monitoramento ambiental a ser realizado através de sensores de movimento, sensores de gás, umidade e temperatura. Desta forma, a arquitetura pode informar as pessoas portadoras de deficiência ou cuidadores sobre possíveis acidentes que podem ocorrer em um local particular. No entanto, o protótipo inicial é restrito a monitorização ambiental e é incapaz de fazer inferências sobre o comportamento do paciente. [4] adicionou novos componentes à arquitetura $\mathrm{SAHHc}$ a fim de abstrair mais informações sobre um paciente particular e permitir o reconhecimento das expressões faciais de um determinado paciente. Assim, novos componentes foram adicionados com o propósito de monitorar o comportamento de atividades do idoso e reconhecer um possível acidente, por exemplo, uma 
queda. Esta plataforma permite que o cuidador ou familiar possa ter mais informações sobre o estado de um idoso em tempo real.

O nível 0 da arquitetura SAHHc ilustrado na Figura 6, é sempre executada no dispositivo embarcado (Raspberry Pi), onde os dados do acelerômetro e giroscópio são enviados. Esta captura de dados é realizada em tempo de real, mas o reconheci-mento da atividade pode ou não ser executado de acordo com a análise de carga de trabalho. Quando a carga de trabalho é baixa, o reconhecimento da atividade é realizado. Assim, o nível 0 é sempre responsável pela captura dos dados, mas o processamento é realizado apenas em casos onde há poucos idosos nos ambientes a serem monitorados. O nível 1 irá realizar o monitoramento da casa e as atividades de um ou diversos idosos. Devido a possibilidade de vários usuários serem monitorados, este nível conta com um servidor local dotado de maior poder de processamento e de armazenamento, além de realizar todo o controle do sistema. No nível 2, o processamento dos dados é realizado na nuvem quando o servidor local está sobrecarregado, determinando assim uma arquitetura de balanceamento de carga e permitindo que a arquitetura SAHHc possa ser escalável. Este nível pode ser frequentemente usado quando o ambiente é uma clínica ou hospital.

O equilíbrio da carga de trabalho de processamento inicializa a análise para determinar qual o nível deve ser utilizado. Se o nível selecionado é o nível 0, o fluxo de processamento será executado apenas no RaspBerry Pi. Caso contrário, o sistema verifica se o servidor local está sobrecarregado ou não. Se não está sobrecarregado, o processamento irá ocorrer localmente, isto é, o servidor pode receber os dados do RaspBerry Pi por meio do protocolo Network File System (NFS) e então realizar a identificação dos movimentos do idoso. No entanto, se o servidor local está sobrecarregado, o processamento passa para a nuvem, e, assim, equilibra a carga total. A transferência dos dados do acelerômetro e do giroscópio a partir do servidor local para a nuvem é realizado por meio do protocolo SSH File Transfer Protocol (SFTP) que permite uma transferência de informação confiável. Por fim, a conexão com o banco de dados é realizada pelo protocolo Transmission Control Protocol (TCP).

\author{
V. AVALIAÇÃO DE DISCUSSÃO \\ A.Acurácia na Identificação de Quedas \\ Nesse trabalho, por considerar o conjunto de dados obtidos
}

por dois sensores distintos (acelerômetro e giroscópio) paraverificar a utilização de algoritmos de ML na identificação das atividades dos idosos, foram coletados dados de 3 indivíduos distintos, entre 18 e 70 anos. Essa estratégia foi desenvolvida considerando a necessidade de garantir que ambos os dados, tanto do acelerômetro quanto do giroscópio, fossem adquiridos no mesmo instante de tempo (timestamp). Apesar de realizadas consultas em banco de dados públicos, não foram encontrados dados de ambos os sensores em conjunto. Nesse sentido, experimentos foram realizados de modo não intrusivo para a aquisição dos dados das atividades estudadas nesse trabalho (andando, correndo, sentado, deitado, em pé e queda). Os dados coletados correspondem a 15 minutos de cada usuário realizando cada uma das atividades. Por sua vez, os dados foram coletados em um intervalo de tempo correspondente a 20 milissegundos. Assim, no final do experimento, foram adquiridos um conjunto de 135.000 dados de cada atividade, totalizando 810.000 dados. A metodologia utilizada para os experimentos foi a realização da média simples de cada 50 linhas de dados, visto que esse número retorna a média do acelerômetro e giroscópio no intervalo de tempo de 1 segundo.

Inicialmente, analisou-se o desempenho dos algoritmos separadamente por meio de uma técnica de Planejamento e Avaliação de Experimentos. Nesse caso foi o $k$-fold crossvalidation $\operatorname{com} k=10$, sendo $k-1$ para treino e o restante para teste. Assim, é possível gerar estimativa de erro mais precisa, pois a média das estimativas tende a taxa de erro verdadeiro conforme $n$ aumenta, em que geralmente é utilizado para pequenos conjuntos de exemplos.

Desse modo, com a finalidade de facilitar a implementação dos algoritmos selecionados, nosso trabalho utiliza o Framework Waikato Environment for Knowledge Analysis (WEKA) [45], amplamente utilizado por pesquisadores das áreas de ML e Mineração de Dados. O WEKA é uma coleção de algoritmos que podem ser aplicados diretamente a um conjunto de dados ou chamado a partir de seu próprio código Java; contém ferramentas para pré-processamento de dados, classificação, regressão, clustering, regras de associação e visualização. É também adequada para o desenvolvimento de novos sistemas de ML [45]. A Tabela I apresenta os parâmetros utilizados para cada classificador no Framework WEKA. 
Tabela I - Parâmetros utilizados nos classificadores selecionados do Weka

\begin{tabular}{|c|c|c|}
\hline Classificador & Implementação & Parâmetros \\
\hline Naive Bayes & NaiveBayes & $\begin{array}{c}\text { debug = false, displayModelInOldFormat }=\text { false } \\
\text { useKernelEstimator }=\text { false, } \\
\text { useSupervisedDiscretization }=\text { false }\end{array}$ \\
\hline Decision Tree & $\mathrm{J} 48$ & $\begin{array}{l}\text { binarySplits }=\text { false, collapseTree }=\text { true }, \text { confidenceFactor }=0.25, \\
\text { minNumObj }=2, \text { numFolds }=3, \text { reducedErrorPruning }=\text { false }, \\
\text { saveInstanceData }=\text { false, seed }=1, \text { subtreeRaising }=\text { true, unpruned } \\
\quad=\text { false, useLaplace }=\text { false, useMDLcorrection }=\text { true }\end{array}$ \\
\hline kNN & IBK & $\begin{array}{c}\mathrm{KNN}=1 \text {, cross Validate }=\text { false, debug }=\text { false, distanceWeighting } \\
=\text { No distance weighting, meanSquared = false } \\
\text { nearestNeighbourSearchAlgorithm }=\text { LinearNNSearch }-\mathrm{A} \\
\text { "weka.core.EuclideanDistance }-\mathrm{R} \text { first-last", windowSize }=0\end{array}$ \\
\hline SVM & SMO & $\begin{array}{c}\text { buildLogisticModels }=\text { false }, \mathrm{c}=1.0, \text { checksTurnedOff }=\text { false }, \\
\text { debug }=\text { false }, \text { epsilon }=1.0 \mathrm{E}-12, \text { filterType }=\text { Normalize training } \\
\text { data, } \text { kernel }=\text { PolyKernek }-\mathrm{C} 250007-\mathrm{E} 1.0, \text { numFolds }=-1, \\
\text { randomSeed }=1, \text { toleranceParameter }=0.001\end{array}$ \\
\hline MPL & MultiLayerPerceptron & $\begin{array}{c}\text { GUI }=\text { false, autoBuild }=\text { true, batchSize }=100, \text { debug }=\text { false, seed } \\
=0, \text { decay }=\text { false, doNotCheckCapabilities }=\text { false, hiddenLayers }= \\
\text { a, learningRate }=0.3, \text { momentum }=0.2, \text { nominalToBinaryFilter }= \\
\text { true, normalizeNumericClass }=\text { true, numDecimalPlaces }=2 \text {, reset }- \\
\text { true, trainingTime }=500, \text { validationSetSize }=0, \\
\text { validationThreshold }=20\end{array}$ \\
\hline
\end{tabular}

Os resultados demonstram que o algoritmo MLP, para esse conjunto de dados, possibilita uma classificação mais precisa, se comparado com os outros classificadores utilizados para comparação. Isso pode ser visto na Figura 7, a qual apresenta os Boxplots referentes às classificações realizadas. É importante ressaltar que o Boxplot com cor vermelha se refere aos resultados do MLP e exibe a mediana da acurácia superior as alcançadas pelos demais classificadores (os valores das medianas podem ser vistas na Tabela II e a maior encontra-se destacada). Além disso, é possível notar uma menor dispersão dos resultados obtidos pelo MLP, o que demonstra uma maior estabilidade em suas execuções.

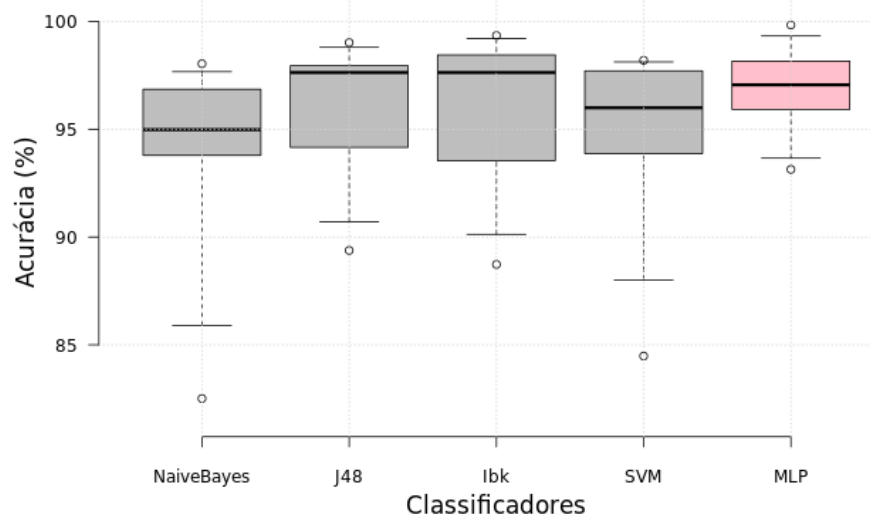

Figura 7: Boxplots das acurácias apresentadas pelos classificadores para determinar a atividade do usuário. Tais resultados foram obtidos com o uso da técnica $k$-fold cross-validation com $\mathrm{k}=10$.
Tabela II - Os valores de acurácia para experimento de identificação da atividade - Melhor precisão foi alcançado pela MLP

\begin{tabular}{cccccc}
\hline & N. Bayes & Dec. Tree & KNN & SVM & MLP \\
\hline test 01 & 90.03 & 92.32 & 88.73 & 93.46 & 93.14 \\
test 02 & 93.46 & 89.38 & 92.64 & 92.32 & 94.29 \\
test 03 & 82.51 & 93.14 & 91.83 & 84.48 & 94.29 \\
test 04 & 94.77 & 98.86 & 99.02 & 96.24 & 97.06 \\
test 05 & 94.93 & 98.53 & 98.53 & 97.71 & 98.70 \\
test 06 & 96.24 & 97.22 & 97.06 & 95.75 & 96.41 \\
test 07 & 98.04 & 99.84 & 99.35 & 97.71 & 99.02 \\
test 08 & 97.06 & 98.53 & 98.86 & 98.03 & 98.20 \\
test 09 & 97.22 & 97.88 & 98.37 & 98.20 & 98.04 \\
test 10 & 95.03 & 97.55 & 97.55 & 95.10 & 97.06 \\
\hline média & 93.93 & 96.33 & 96.19 & 94.90 & $\mathbf{9 6 . 6 2}$ \\
\hline
\end{tabular}

Com objetivo de validar tais resultados, foram realizadas diversas análises estatísticas. Inicialmente, utilizamos o método de Shapiro Wilk para verificar sua adequação à normalidade e consequentemente conduzir para testes paramétricos ou nãoparamétricos. Conforme os valores- $p$ apresentados na Tabela III são todos maiores que 0.05 , consideramos aceita a hipótese de normalidade com confiabilidade de 95\%. Portanto, utilizaremos testes paramétricos para as próximas análises. 
Tabela III - Valores- $p$ dos conjuntos de resultados.

\begin{tabular}{cc}
\hline Classificadores & $\begin{array}{c}\text { Shapiro } \\
\text { Wilk }\{\text { Valor }-\mathrm{p}\}\end{array}$ \\
\hline Naive Bayes & 0.89240 \\
Árv. Dec. & 0.07236 \\
kNN & 0.52510 \\
SVM & 0.65000 \\
MLP & 0.66320 \\
\hline
\end{tabular}

As comparações de pares realizadas com o teste $t$ são exibidas na Tabela IV. Tais resultados mostram que o MLP não apresenta diferença estatisticamente significativa com os demais classificadores. Contudo, dado a acurácia mediana superior e a menor dispersão desses resultados apresentados pelo MLP, consideramos tal abordagem mais eficiente do que o uso dos demais classificadores para a identificação da atividade do usuário.

Tabela IV - Comparações pareadas utilizando testes $t$.

\begin{tabular}{ccccc}
\hline & N.B. & Árv. & kNN & SVM \\
\hline Árv. & 1.0 & - & - & - \\
kNN & 1.0 & 1.0 & - & - \\
SVM & 1.0 & 1.0 & 1.0 & - \\
MLP & 0.8 & 1.0 & 1.0 & 1.0 \\
\hline
\end{tabular}

Estes resultados demonstram que, entre os classificadores que foram explorados para esse contexto, o MLP é a técnica que tem uma maior precisão na classificação das atividades de acordo com os sensores utilizados (acelerômetro e giroscópio). A Tabela $\mathrm{V}$ mostra a matriz de erro, também conhecida como a matriz de confusão, que considera a classificação das atividades dos usuários. Neste quadro, as colunas representam os dados de referência, enquanto que as linhas representam a classificação gerada a partir da técnica de classificação da atividade.

Tabela V - Matriz de confusão em relação à classificação do MLP

\begin{tabular}{ccccccc}
\hline & $\mathrm{A}$ & $\mathrm{B}$ & $\mathrm{C}$ & $\mathrm{D}$ & $\mathrm{E}$ & $\mathrm{F}$ \\
\hline $\begin{array}{c}\text { Andando }= \\
\text { A }\end{array}$ & $\mathbf{1 0 1 7}$ & 0 & 1 & 0 & 2 & 0 \\
Correndo $=$ & 0 & $\mathbf{1 0 0 9}$ & 0 & 5 & 2 & 4 \\
$\mathrm{~B}$ & & & & & & \\
Deitado $=\mathrm{C}$ & 0 & 0 & $\mathbf{1 0 2 0}$ & 0 & 0 & 0 \\
Em Pé $=\mathrm{D}$ & 0 & 17 & 0 & $\mathbf{9 7 2}$ & 5 & 20 \\
Queda $=\mathrm{E}$ & 12 & 1 & 13 & 9 & $\mathbf{9 8 1}$ & 4 \\
Sentado $=\mathrm{F}$ & 0 & 5 & 0 & 35 & 1 & $\mathbf{9 7 9}$ \\
\hline
\end{tabular}

\section{A.Desempenho Computacional da Arquitetura}

Outro fator importante que deve ser analisado é o desempenho da proposta na arquitetura SAHHc. Essa análise permite saber o comportamento esperado da arquitetura e investigar maneiras eficientes de novas implementações para evitar sobrecarregar os nós da rede e prevenir possíveis gargalos.

Nesse sentido, dois cenários foram definidos para as experiências seguintes empregando a metodologia proposta em diferentes elementos de rede: i.) processamento no sensor embarcado, e ii.) processamento no Nó Decisor. No primeiro cenário, onde a identificação e classificação das atividades do idoso é executado nos nós de sensores, os resultados permitem saber o intervalo mínimo entre cada informação é capturado, uma vez que antes de capturar uma nova informação, é necessário o processamento da última informação capturada e transmissão dos resultados ao Nó Decisor. No entanto, esta abordagem restringe o desempenho porque os dispositivos de sensores têm recursos muito limitados. No segundo cenário, os nós sensores somente ficam responsáveis por capturar as informações e transmiti-las ao Nó Decisor, que é responsável pelo processamento de todas as informações e tomadas de decisões apropriadas. Embora o Nó Decisor tenha mais recursos computacionais disponíveis, uma vez que existem vários nós de sensores, o Nó Decisor fará o processamento competitivo e pode ser sobrecarregado, se a carga de trabalho é muito pesada.

Assim, para que as comparações do desempenho possam ser mais justas, foi assumido que as informações já estão armazenadas nos elementos que empregam a metodologia. Desta forma, os tempos de operação para cada experiência pode ser determinada para medir o nível de carga e permitir a sua comparação. Nesse sentido, 30 repetições foram realizadas para cada configuração em intervalos de 10 segundos para conseguir uma melhor análise estatística dos resultados.

Os experimentos foram planejados para simular situações da vida real mais de perto e representam o comportamento da arquitetura nestas situações. Assim, no primeiro cenário (incorporados - Nível 0), em que os nós sensores processam as informações adquiridas, não há competição para o processamento (é assumido que os nós não são empregados em qualquer outra aplicação), e independentemente do número de nós sensores presente na arquitetura; também é possível assumir que o tempo de execução é igual para todos os nós (uma vez que todos os nós sensores têm o mesmo microprocessador). Por outro lado, no segundo cenário (no servidor local, o elemento responsável pelas decisões captadas pelos nós de sensores - Nível 1), pode haver competição para processamento, com um nível máximo igual ao número de nós sensores que compõem a arquitetura. No pior cenário possível, onde as informações são recebidas ao mesmo tempo e as várias instâncias da metodologia proposta são iniciadas imediatamente, $8,16,32$ e 64 foram tomados como o número de informações em processamento simultâneo.

A Figura 8 exibe os tempos de execução obtidos para cada configuração experimental. Embora a configuração OnBoard (no nó sensor) não tenha concorrência para o processamento, esta abordagem teve o maior tempo de execução de todos. Isto é devido aos recursos computacionais limitados nos gânglios sensor (nas experiências foi considerada uma arquitetura com o modelo de Raspberry Pi B para os nós sensores). Raspberry Pi modelo B possui processador ARM (1 core - $700 \mathrm{Mhz}$ de Clock), 512 MB de RAM e cartão SD de 8GB. O Nó Decisor está hospedado em um servidor local equipado com um processador Intel i5 (4 núcleos [dois físicos e dois por HyperThreading] - $3 \mathrm{GHz}$ de Clock), 16 GB de RAM e um disco de 1 TB. 


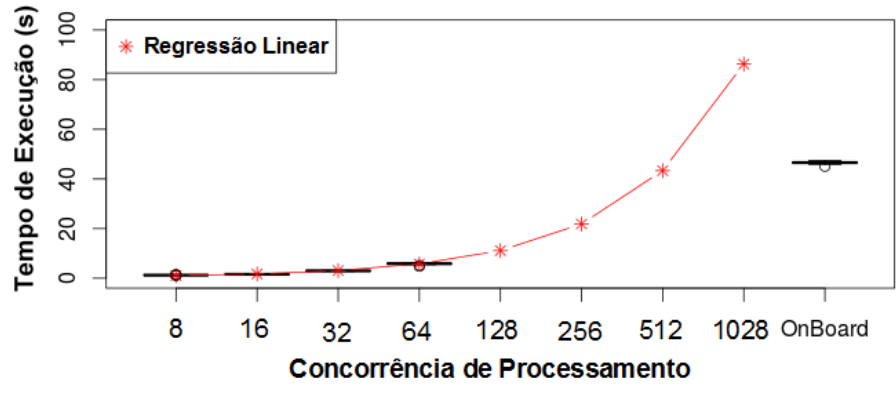

Figura 8: Utilização de regressão linear para determinar o processamento em dois cenários distintos.

Os resultados da implementação no Nó Decisor podem ser vistos nos primeiros 4 pontos da Figura 8. Estes resultados também se referem ao nível de concorrência em cada experimento. Pode ser visto que o tempo de execução cresce, como o nível de aumento da concorrência. No entanto, apesar da experiência fornecer somente para 64 processos simultâneos, o maior tempo de execução observada no Nó Decisor é cerca de 5 vezes mais curto do que o tempo de execução da experiência no modo Onboard.

Com base nestes resultados foi realizada uma regressão linear e é utilizado para inferir o nível de concorrência necessária para o tempo de execução, se o Nó Decisor se torna maior do que o OnBoard, que sugere uma saturação da arquitetura. Pode-se pressupor que as características do aumento do tempo de execução são mantidas enquanto os cálculos são feitos para novos níveis de competição. Estes resultados são indicados por asteriscos na Figura 8 com uma linha reta entre os pontos em vermelho. Assim, verificou-se que, quando existe uma competição envolvendo 512 informações, o tempo de execução é próximo daquele apresentado pelo modo Onboard, mas ainda mais baixa. Quando o nível de competição é igual a 1028 informações, pode-se observar que a saturação da arquitetura tem um tempo de execução de cerca de duas vezes tão elevada se comparado ao cenário Onboard. Isto torna possível assumir que a execução da metodologia proposta no elemento Nó Decisor é viável com arquiteturas que têm até 512 elementos sensores. Se for necessário um maior número de elementos de sensor, uma abordagem que envolve o uso de cenários somente Onboard, será mais apropriado.

A.Análise dos efeitos de transferência das informações para processamento na nuvem

Uma estratégia alternativa que a arquitetura SAHHc pode adotar é utilizar a nuvem para executar a identificação da atividade do idoso (Nível 2). Neste caso, a arquitetura não é afetada pela sobrecarga ao processar as informações captadas pelos elementos sensores. Por outro lado, a transferência das informações para processamento na nuvem pode causar congestionamento na transmissão e, portanto, aumentar o tempo necessário para o envio de mensagens.

O conceito de computação da nuvem, do inglês Cloud Computing, é de um sistema que possui recursos computacionais elásticos e pode, assim, manter as característi- cas necessárias para um dado serviço. Em vista disto, pode-se assumir que o tempo de processamento pode ser ajustado de acordo com a aplicação atual. No entanto, o tempo para enviar os dados não faz parte das características dos serviços contratados, pois eles dependem de recursos que não são controlados pelo provedor de Cloud Computing.

O tempo de transmissão é parte do tempo total (que inclui o tempo de aquisição da informação até ao fim do procedimento metodológico proposto) e resulta em um aumento no intervalo mínimo entre os processos de monitoramento. Com isto em mente, avaliamos o impacto das transmissões simultâneas que resultaram em um aumento do intervalo mínimo entre os processos de monitoramento. Por esta razão, foi conduzido um experimento para medir a transmissão simultânea das informações obtidas pelos sensores (acelerômetro e giroscópio). Este foi realizada através da ferramenta de comunicação SFTP. A ligação para a comunicação foi estabelecida entre o Nó Decisor e um servidor virtual remoto implementado na nuvem.

O servidor real é um Dell PowerEdge R720, Intel Xeon 32 núcleos (dois processadores físicos com oito núcleos e Hyper Threading (2x)) - $2 \mathrm{GHz}$ de Clock, $256 \mathrm{~GB}$ de RAM e 6 HDs SAS hot-plug 3,5 de 600 GB em RAID 5. A máquina virtual foi hospedada neste servidor real e usado 4 VCPUs, 16GB de RAM e 20 GB de disco.

O processo de transmissão das informações segue o mesmo esquema de paralelismo conduzida nas experiências anteriores, isto é, 8, 16, 32 e 64 informações são enviadas através da rede simultaneamente. O tempo decorrido para o envio desta quantidade de informações em paralelo pode ser visto na Figura 9.

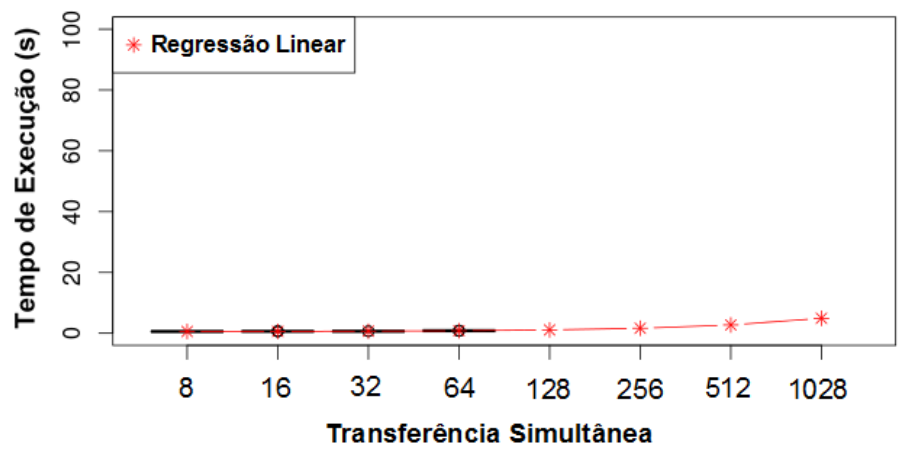

Figura 9: Usando a regressão linear para demonstrar o aumento do tempo de transmissão simultânea dos dados do servidor local para o servidor virtual na nuvem.

De acordo com o que é mostrado na Figura 9, o tempo de transferência aumenta linearmente, de acordo com o aumento do paralelismo. Em vista disto, uma regressão linear foi utilizada para estimar o tempo de transferência para 128, 256, 512 e 1028 informações. Pode-se dizer que o tempo total de processamento não excedeu o tempo de processamento realizado pelo nosso modo OnBoard. Este foi, no caso de (a) se uma infraestrutura de nuvem virtual é utilizada para obter um tempo de resposta similar para processamento de 256 
informações simultaneamente (Figura 8), ou (b) se usa um servidor com uma semelhante configuração para o elemento Nó Decisor. Isso nos ajudará a evitar a saturação da arquitetura que é implementado para o processamento de 1028 informações. A razão para isto é que o servidor irá adaptar os seus recursos de computação para manter o tempo de resposta combinado.

\section{CONCLUSÕES}

Este trabalho explorou a utilização de dados de sensores para detectar a queda de pacientes idosos. Isso pode fornecer um importante auxílio no cuidado da saúde em ambientes domésticos inteligentes, de forma automática, através de uma infraestrutura de IoT (ou seja, sem a necessidade de intervenção humana para detectar novos recursos). Fizemos uso dos dados combinados do acelerômetro e giroscópio com o intuito de aumentar a precisão na identificação das atividades dos idosos. Os experimentos foram conduzidos nesta frente e obteve uma precisão de $96.621 \%$ ao identificar as atividades executadas pelo paciente (andando, correndo, sentado, deitado, em pé e queda).

Nossa proposta demonstra que as pessoas podem ter o seu próprio sistema de saúde individual ou submetidos a tratamento especializado. Os experimentos foram conduzidos para mostrar a adequação de nossa abordagem na arquitetura SAHHc, que além de ser proposta para o cuidado de idosos, também pode ser aplicada no cuidado de pessoas com alguma deficiência ou mobilidade reduzida. Com isso, adotamos uma abordagem que levou em consideração todas essas características e experiências realizadas para validar a ideia em termos de desempenho, precisão e análise estatística.

Como pesquisa futura pretendemos explorar outros sensores para detecção de quedas de idosos, como por exemplo a utilização de imagens. Isso pode ser novamente um bom desafio para o SAHHc, uma vez que vai exigir ciclos de processamento e comunicações, juntamente com abordagens evolutivas em um ambiente heterogêneo. Além disso, vale mencionar que pretendemos melhorar o monitoramento dos idosos por meio de outros sensores, tais como como microfone e sensor de presença, com o objetivo de prevenir acidentes em ambientes domiciliares.

\section{REFERÊNCIAS}

[1] Gonçalves, Vinícius Pereira, et al. "An Analitic Approach to Evaluate Flexible Mobile User Interfaces for the Elderly." ICEIS (3). 2012.

[2] Gonçalves, Vinícius P., et al. "Providing adaptive smartphone interfaces targeted at elderly people: an approach that takes into account diversity among the elderly." Universal Access in the Information Society (2015): $1-21$.

[3] Camargos, Mirela Castro Santos, Roberto Nascimento Rodrigues, and Carla Jorge Machado. "Idoso, família e domicílio: uma revisão narrativa sobre a decisão de morar sozinho." Rev bras estud popul 28.1 (2011): 217-230.

[4] Mano, Leandro Y., et al. "Exploiting IoT technologies for enhancing Health Smart Homes through patient identification and emotion recognition."Computer Communications (2016).

[5] Omatu, Sigeru, et al., eds. Distributed Computing, Artificial Intelligence, Bioinformatics, Soft Computing, and Ambient Assisted Living: 10th International Work-Conference on Artificial Neural Networks, IWANN 2009 Workshops, Salamanca, Spain, June 10-12, 2009. Proceedings. Vol. 5518. Springer, 2009.
[6] Stankovic, J. A., et al. "Wireless sensor networks for in-home healthcare: Potential and challenges." High confidence medical device software and systems (HCMDSS) workshop. 2005.

[7] Rialle, Vincent, et al. "Health" smart" home: information technology for patients at home." Telemedicine Journal and E-Health 8.4 (2002): 395409.

[8] Russek, Linda G. "Alarm for patient monitor and life support equipment system." U.S. Patent No. 5,319,355. 7 Jun. 1994.

[9] Sui, Yongkun, Chanmin Ahn, and Chong H. Ahn. "A new smart falldown detector for senior healthcare system using inertial microsensors." 2014 36th Annual International Conference of the IEEE Engineering in Medicine and Biology Society. IEEE, 2014.

[10] Intel Corporation, "Internet-of-Things (IoT) Advances Home Healthcare for Seniors," http://goo.gl/aFye65, 2015.

[11] Kühner, Daniel. "'" Internet der Dinge Telekommunikationsinfrastruktur."Seminarband: Mobile und Verteilte Systeme-Ubiquitous Computing Teil IV. Seminarband: Mobile und Verteilte Systeme-Ubiquitous Computing Teil IV. Universität KarlsruheFakultät für Informatik (2007): 1-15.

[12] Aarts, Emile, and Frits Grotenhuis. "Ambient intelligence 2.0: Towards synergetic prosperity." Journal of Ambient Intelligence and Smart Environments 3.1 (2011): 3-11.

[13] Peña-López, Ismael. "ITU Internet report 2005: the internet of things." (2005).

[14] Dohr, Angelika, et al. "The Internet of Things for Ambient Assisted Living."ITNG 10 (2010): 804-809.

[15] Gubbi, Jayavardhana, et al. "Internet of Things (IoT): A vision, architectural elements, and future directions." Future Generation Computer Systems 29.7 (2013): 1645-1660.

[16] Takács, Barnabás, and Dávid Hanák. "A mobile system for assisted living with ambient facial interfaces." IADIS Int. J. Comput. Sci. Inf. Syst 2 (2007): 33-50.

[17] Riva, Giuseppe. "Ambient intelligence in health care." CyberPsychology \& Behavior 6.3 (2003): 295-300.

[18] Geraldo Filho, P. R., et al. "An intelligent approach for improving energy efficiently in smart grids." Proceedings of the International Conference on Wireless Networks (ICWN). The Steering Committee of The World Congress in Computer Science, Computer Engineering and Applied Computing (WorldComp), 2013.

[19] Geraldo Filho, P. R., et al. "ResiDI: An Intelligent Decision Platform for Residential Infrastructure Using Wireless Sensors and Actuators." Computer Networks and Distributed Systems (SBRC), 2015 XXXIII Brazilian Symposium on. IEEE, 2015.

[20] Augusto, Juan Carlos, et al. "Enhanced healthcare provision through assisted decision-making in a smart home environment." 2nd Workshop on Artificial Inteligence Techniques for Ambient Inteligence. 2007.

[21] Ray, Partha P. "Home Health Hub Internet of Things (H 3 IoT): An architectural framework for monitoring health of elderly people." Science Engineering and Management Research (ICSEMR), 2014 International Conference on. IEEE, 2014.

[22] Helal, Sumi, et al. "The Gator Tech Smart House: A Programmable Pervasive Space." The Engineering Handbook of Smart Technology for Aging, Disability, and Independence (2008): 693-709.

[23] Abowd, Gregory D., et al. "The aware home: A living laboratory for technologies for successful aging." Proceedings of the AAAI-02 Workshop "Automation as Caregiver. 2002.

[24] Kunze, Christophe, et al. "Kontextsensitive Technologien und Intelligente Sensorik für Ambient-Assisted-Living-Anwendungen." Ambient Assisted Living-AAL (2008).

[25] EU Commission. "Communication from the commission to the European parliament, the council, the European economic and social committee and the committee of the regions: Strategic guidelines for the sustainable development of EU aquaculture COM (2013) 229 final." (2013).

[26] Banerjee, Tanvi, et al. "Recognizing complex instrumental activities of daily living using scene information and fuzzy logic." Computer Vision and Image Understanding 140 (2015): 68-82.

[27] Bonomi, Alberto G., et al. "Improving assessment of daily energy expenditure by identifying types of physical activity with a single accelerometer." Journal of Applied Physiology 107.3 (2009): 655-661.

[28] O'Donovan, Karol, and Steve Ayer. "Real-time joint angle measurement using the shimmer wireless sensor platform." Proceedings of the First ACM Workshop on Mobile Systems, Applications, and Services for Healthcare. ACM, 2011. 
[29] M. A. System. (2015) QMedic - medical alert system - full review. [Online]. Available: http://medicalalertsystemreviews.net/qmedicfullreview/

[30] ] Michalski, Ryszard S., Jaime G. Carbonell, and Tom M. Mitchell, eds.Machine learning: An artificial intelligence approach. Springer Science \& Business Media, 2013.

[31] Bailenson, Jeremy N., et al. "Real-time classification of evoked emotions using facial feature tracking and physiological responses." International journal of human-computer studies 66.5 (2008): 303-317.

[32] Yuan, Gu, et al. "A GMM based 2-stage architecture for multi-subject emotion recognition using physiological responses." Proceedings of the 1 st Augmented Human International Conference. ACM, 2010.

[33] Faceli, Katti, et al. "Inteligência Artificial: Uma abordagem de aprendizado de máquina." Rio de Janeiro: LTC (2011).

[34] Peter, Christian, and Bodo Urban. "Emotion in human-computer interaction."Expanding the Frontiers of Visual Analytics and Visualization. Springer London, 2012. 239-262.

[35] Duda, Richard O., and Peter E. Hart. Pattern classification and scene analysis. Vol. 3. New York: Wiley, 1973.

[36] Ramakrishnan, S., and Ibrahiem MM E1 Emary. "Speech emotion recognition approaches in human computer interaction. "Telecommunication Systems52.3 (2013): 1467-14783.

[37] Chanel, Guillaume, et al. "Short-term emotion assessment in a recall paradigm." International Journal of Human-Computer Studies 67.8 (2009): 607-627.
[38] Littlewort, Gwen, et al. "The computer expression recognition toolbox (CERT)." Automatic Face \& Gesture Recognition and Workshops (FG 2011), 2011 IEEE International Conference on. IEEE, 2011.

[39] Clark, Peter, and Tim Niblett. "The CN2 induction algorithm." Machine learning 3.4 (1989): 261-283.

[40] Fanucchi, RODRIGO Z., M. A. R. I. O. Oleskovicz, and D. A. N. I. E. L. Barbosa. "Análise da Detecção de Faltas de Alta Impedância Utilizando Redes Neurais Artificiais com Topologias Baseadas em Perceptron Multicamadas e Redes RBF." (2013): 1-6.

[41] Giacomossi, L. C., et al. "Classificação de Movimentos Normais e Anormais Capturados por Acelerômetro: Resultados Preliminares." $V$ Latin American Congress on Biomedical Engineering CLAIB 2011 May 16-21, 2011, Habana, Cuba. Springer Berlin Heidelberg, 2013.

[42] Kruse, Rudolf, et al. "Multi-layer perceptrons." Computational Intelligence. Springer London, 2013. 47-81.

[43] Mano, Leandro Y., et al. "Exploiting the Use of Ensemble Classifiers to Enhance the Precision of User's Emotion Classification." Proceedings of the 16th International Conference on Engineering Applications of Neural Networks (INNS). ACM, 2015.

[44] Freitas, Diulie J., et al. "Combining cell phones and WSNs for preventing accidents in smart-homes with disabled people." 2015 7th International Conference on New Technologies, Mobility and Security (NTMS). IEEE, 2015.

[45] Bouckaert, Remco R., et al. "WEKA Manual for Version 3-78." Hamilton, New Zealand (2013). 